\title{
miR-I39 Controls Viability Of Ovarian Cancer Cells Through Apoptosis Induction And Exosome Shedding Inhibition By Targeting ATP7A
}

This article was published in the following Dove Press journal: OncoTargets and Therapy

\author{
Fang Xiao \\ Songshu Xiao \\ Min Xue \\ Department of Obstetrics and \\ Gynecology, Third Xiangya Hospital of \\ Central South University, Changsha, \\ Hunan 4I00I3, People's Republic of \\ China
}

Background: Emerging proof suggests that microRNA (miRNA) malfunction is correlated to the generation and development of multiple malignancies. It has been proven that miRNA (miR)-139 represses a variety of malignancies. However, the understanding of its impact on ovarian cancer (OC) is insufficient. We aimed to investigate the expression and function of miR-139 in OC.

Methods: The expression of mir-139 was detected and recorded, and the relationship of mir139 remaining OC cells was explored. At the same time, we studied the correlation between ATP7A and mir-139 by the luciferase reporter test, Western blot, and quantitative real-time reverse transcription PCR.

Results: The expression of miR-139 was remarkably downregulated in OC specimens. Furthermore, excessive miR-139 expression noticeably inhibited the migration, colony generation, proliferation, and invasion of OC cells. In addition, excessive miR-139 expression remarkably repressed the death and the expression of proteins related to cell death in $\mathrm{OC}$ cells, as well as inhibited the shedding of exosomes. According to the luciferase reporter test, Western blot, and quantitative real-time reverse transcription PCR, miR-139 directly targeted ATP7A. Furthermore, the expression of ATP7A was found to be negatively related to miR139 levels in OC specimens. It was revealed via a rescue experiment that excessive ATP7A expression counteracted the repressive effect of miR-139 in OC cells.

Conclusion: It was revealed via an in vivo study that miR-139 remarkably inhibited the growth of malignancies by downregulating ATP7A in nude mice. miR-139 represses the development of malignancies in OC by directly targeting ATP7A, offering an innovative approach for molecular therapy of OC.

Keywords: apoptosis, ATP7A, exosome shedding, miR-139, ovarian cancer

\section{Introduction}

Ovarian cancer (OC) has a high mortality rate worldwide. ${ }^{1}$ Despite huge progress in OC therapy, the 5-year overall survival for OC patients is $35 \%$ to $38 \%$, which can be mainly attributed to diagnosis at terminal stage, frequent metastasis, and the fast generation of chemoresistance. ${ }^{2-5}$ Consequently, elucidating the etiology of the illness and discovering crucial agents that can assist in the diagnosis and innovative therapy of OC are necessary.

MicroRNAs (miRNAs) are a group of small endogenous RNAs without coding functions that consist of 18 to 22 nucleotides and inhibit the expression of target genes via direct binding with the 3 'untranslated regions (3'-UTRs) of their mRNA, thereby
Correspondence: Min Xue Gynecology, Third Xiangya Hospital of Central South University, No. 138,

Tongzipo Road, Yuelu District, Hexi, Changsha, Hunan 410013, People's

Republic of China

Tel +86-I3687354678

Email xuemmm678@163.com 
downregulating gene expression both at the mRNA and protein levels. ${ }^{6}$ Previous research has proven that miRNAs contribute to the modulation of multiple malignancies and thus offer promising treatment options. ${ }^{7-9}$ It has been demonstrated that some miRNAs can malfunction, such as miR-985p, miR-92, miR-141, and miR-93-5p, and result in the generation and development of OC. ${ }^{10-13}$ It was revealed that miR-139, which modulates various cellular processes including invasion, cell cycle, proliferation, and vessel generation, malfunctions in some kinds of malignancies. ${ }^{14-17}$ Emerging proofs about colorectal cancer, hepatocellular carcinoma, bladder cancer, non-small cell lung cancer, breast cancer, oral tongue squamous cell carcinoma, and esophageal squamous cell carcinoma have indicated that miR-139 suppresses malignancies and is thus commonly downregulated in malignancies. ${ }^{14,18-22}$

Through mediating Wnt/ $\beta$-catenin signaling pathway, miR-139 is involved in the cell growth and metastasis of bladder cancer cells. ${ }^{12}$ By direct targeting NOB1, miR-139 can induce the cell apoptosis and inhibit the metastasis of cervical cancer cells. ${ }^{13}$ In glioblastoma multiforme, miR139 can hinder the cell migration and invasion of cancer cells through targeting ZEB1/2.

Copper-transporting ATPases are intracellular transporters that maintain cellular copper homeostasis. Dysregulated expression of ATP7A in ovarian cancer cells can alter the amount of cellular copper, which could then impact the activities of other transporters that import or export platinum derivatives. Increased expression of ATP7A in ovarian cancer cells predicts short survival for patients treated with platinum derivatives and suggests a role for ATP7A in OC. Whether miR-139 regulated ATP7A in ovarian cancer cells, thus to affect the cell proliferation and apoptosis? This remains to be studied. Consequently, our research aimed to explore miR-139 expression in clinical OC specimens and OC cell lines to elucidate its molecular mechanism and impact on OC development and metastasis.

\section{Materials And Methods}

\section{Clinical Specimens}

Biopsy specimens of OC and surrounding non-malignant tissues were obtained from 31 patients treated at our hospital. The surrounding tissues were $3 \mathrm{~cm}$ away from the malignancy fringe with no obvious malignant cells, as assessed by an expert pathologist. All specimens were immediately frozen at $-80^{\circ} \mathrm{C}$ after surgery using liquid nitrogen. No patients had undergone radiation therapy or chemotherapy prior to surgery. Fully informed consent was acquired from every participant, which won approval from Research Ethics Committee of our hospital.

\section{Cell Culture And Transfection}

Human OC cell lines HO-8910 and SKOV3 were purchased from Tumor Cell Bank of the Chinese Academy of Medical Science (Beijing, P.R. China). RPMI-1640 medium with $10 \%$ FBS was used and incubated at $37^{\circ} \mathrm{C}$ with $5 \% \mathrm{CO}_{2}$.

MiR-139 mimics, inhibitor, and negative control (NC) mimics were purchased from GenePharma (Shanghai, P.R. China). ATP7A overexpression plasmids (pCDNA3.1-ATP7 A) were purchased from the same company. SKOV3 cells were seeded in 24-well plates and were transfected with vectors $(5 \mu \mathrm{g})$ or miRNA mimics/NC $(100 \mathrm{mM})$ using Lipofectamine 2000 in serum-free media.

\section{RNA Isolation And Quantitative Real-Time Reverse Transcription PCR (qRT-PCR)}

TRIzol reagent was used to isolate total RNA from the cultivated cells or tissues as instructed. SmartSpec Plus spectrophotometer was used for RNA quantification and quality check by purity assessment based on A260/A280 ratio. SYBR PrimeScript miRNA RT PCR Kit was used to measure the expression of miR-139-5p. The corresponding primers of miR-139-5p and U6 were acquired from Applied Biosystems. Two micrograms of total RNA underwent reverse transcription (RT) to generate cDNA with the help of PrimeScript RT Reagent Kit using oligo (dT) primers (Takara). SYBR Green Real-Time Master Mix was used for qRT-PCR. GAPDH served as an internal control. The expression levels of ATP7A mRNA and miR139 were normalized against GAPDH or U6 levels based on the $2^{-\Delta \Delta \mathrm{Ct}}$ approach.

\section{Luciferase Reporter Assay}

Wild type (WT) ATP7B or ATP7A luciferase reporter gene vectors (called ATP7B-WT-3'UTR or ATP7A-WT-3' UTR), as well as mutant ones were constructed. HEK293T cells were co-transfected with the corresponding vectors and miR-139 inhibitors or mimics, and then cultured overnight in 24-well plates. Dual Luciferase Reporter Assay (Promega, USA) was performed 48 $\mathrm{h}$ after transfection. 


\section{Separation Of Exosomes}

Exosomes were separated using differential centrifugation approaches. Cultures were centrifuged and the supernatant was then centrifuged at $2000 \times g$ for $20 \mathrm{~min}$ to acquire apoptotic bodies, and then at $12,200 \times g$ for $60 \mathrm{~min}$ to harvest the microvesicles. Another supernatant was passed through $0.22-\mu \mathrm{m}$ filters and was centrifuged at 120,000 $\times g$ for $2 \mathrm{~h}$ to obtain the exosomes. One milliliter of TRIzol reagent was added to every tube of acquired pellet to isolate total RNA as per the manufacturer's instructions.

\section{Proliferation Test}

Cell Counting Kit-8 was used to assess cell proliferation. In brief, cells were cultured in 96-well plates. CCK-8 reagent was added to the corresponding wells, and allowed to incubate for $2 \mathrm{~h}$. Media with CCK-8 were subsequently transferred to new 96-well plates and absorbance was measured.

\section{Cell Cycle Test}

Cells went through 12-h starvation to synchronize the cultures prior to 24 -h re-activation with $10 \%$ FBS. Cells were fixed and FACS Caliber flow cytometer was subsequently used to categorize the cells. Flowjo software (Treestar Inc., USA) was used to evaluate the cell phase distribution.

\section{Colony Generation Test}

Cells were incubated with $0.25 \%$ trypsin. Nearly five hundred cells were seeded in 6-well plates ( 250 cells/ $\mathrm{mL})$. Cells were fixed for $10 \mathrm{~min}$ using anhydrous ethanol and stained for $30 \mathrm{~min}$ with $0.1 \%$ crystal violet. Colonies comprising 50 cells were counted and the relative colony number was acquired. Colony-generating capability was assessed by obtaining the ratio of the number of colonies generated in the transfection group to that in the control group multiplied by 100 .

\section{Flow Cytometry (FC)}

PBS was used to wash the acquired cells. A million cells were isolated from each specimen, and stained with an Annexin V-FITC/propidium iodide (PI) kit. FC (BD FACS Aria; BD Biosciences, Franklin Lakes, NJ) was then performed to detect positive cells $48 \mathrm{~h}$ after transfection.

\section{Cell Migration Test}

Transwell test was performed to examine cell migration. In brief, $5 \times 10^{4}$ cells were suspended in DMEM without serum and were seeded on the top well of 24-well poly- carbonate transwell filters. DMEM with 10\% FBS was supplemented to the bottom well. Cells at the top surface were scraped off after a 24-h incubation and those at the bottom surface were fixed, stained, and quantified.

\section{Cell Invasion Test}

Twenty-four-well transwell chambers with $8-\mu \mathrm{m}$ pore size polycarbonate membranes were used for the invasion test. Transwell chambers initially coated with Matrigel were seeded with cells. Cells suspended in $200 \mu \mathrm{L}$ of DMEM without serum were seeded on the top chamber, while those in $8 \mu \mathrm{L}$ of DMEM with $10 \%$ FBS were seeded on the bottom chamber. Cells that did not undergo invasion were eliminated from top chamber using a cotton swab after $24 \mathrm{~h}$ of incubation, and those that invaded the bottom were fixed and stained with $0.1 \%$ crystal violet. Cells were quantified in 6 random fields for every well. Relative folds of cells with invasion were displayed.

\section{Xenograft Malignancy Model}

BALB/c mice were purchased from Peking Union Medical College (Beijing, China) and reared in sterile conditions. Mice received subcutaneous injection of SKOV3 cells $\left(5 \times 10^{6}\right.$ per $\left.0.1 \mathrm{~mL}\right)$ through their back. The volume and weight of the mice were examined. The volume of the malignancies of the $\mathrm{NC}$ or miR-139 groups ( $\mathrm{n}=6$ each) were measured every 3 days based on the following formula: volume $=0.5 \times$ length $\times$ width $\times$ width. Mice were sacrificed 21 days subsequent to injection, and the malignancies were excised and prepared. All mice were treated in accordance with protocols approved by Institutional Animal Care and Use Committee of Third Xiangya Hospital of Central South University, and the committee approved the experiment.

\section{Western Blot (WB)}

Lysates were homogenized using a lysis buffer (Beyotime, China), while Bradford assay was used for protein quantification. Proteins were assessed via standard SDS-PAGE. Various concentrations $(15 \%-80 \%)$ of Tris- $\mathrm{HCl}$ polyacrylamide gels (Bio-Rad) were used to isolate proteins, which were then transferred onto PVDF membranes. After blocking, blots were incubated with primary antibodies, including anti-ATP7A, anti-Bax, anti- $\beta$-actin, and anti-Bcl2 antibodies. Another incubation was carried out with secondary antibodies. Enhanced chemiluminescence (ECL) plus detection reagent (Pierce, Rockford, IL, USA) was used to examine immunoreactive bands. 


\section{Statistical Analysis}

Results were displayed as mean \pm SEM. Differences between various groups were evaluated using two-tailed, unequal-variance Student's $t$-test or ANOVA prior to Tukey's post hoc analysis. A $p$-value $<0.05$ was regarded to be statistically significant.

\section{Results}

\section{miR-I39 Was Downregulated In OC Specimens}

Our research explored miR-139 expression in 31 OC specimens matched to normal specimens to reveal the impact of miR-139 on the generation and development of OC. MiR-139 was found to be downregulated in OC specimens compared to control specimens (Figure 1). Moreover, both transcription and translation of ATP7A were remarkably increased in OC specimens in comparison with those in the control specimens. These findings indicate that the expression of miR-139 was inhibited in OC and that it potentially modulated ATP7A.

\section{miR-I39 Directly Targeted ATP7A To Impair Exosome Secretion-Modulated miR-I39 Trafficking In SKOV3 Cells}

Bioinformatics analysis indicated that miR-139 targeted ATP7A. Excessive miR-139 expression impaired ATP7A 3'UTR function in SKOV3 cells, while miR-139 inhibition improved it (Figure 2A and B). Consequently, miR-139 regulated ATP7A expression probably via direct 3'-UTR binding. Moreover, excessive miR-139 expression inhibited ATP7A translation. In contrast, miR-139 inhibition increased ATP7A

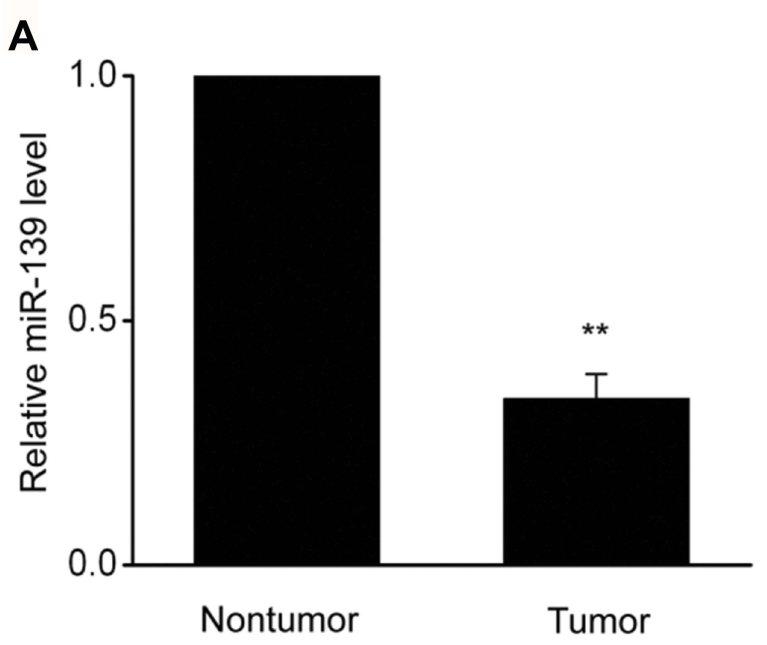

C

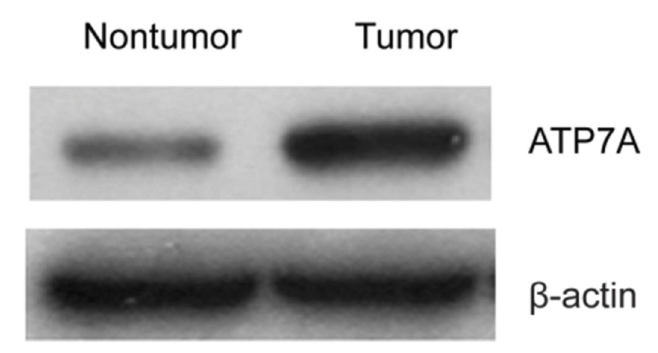

B

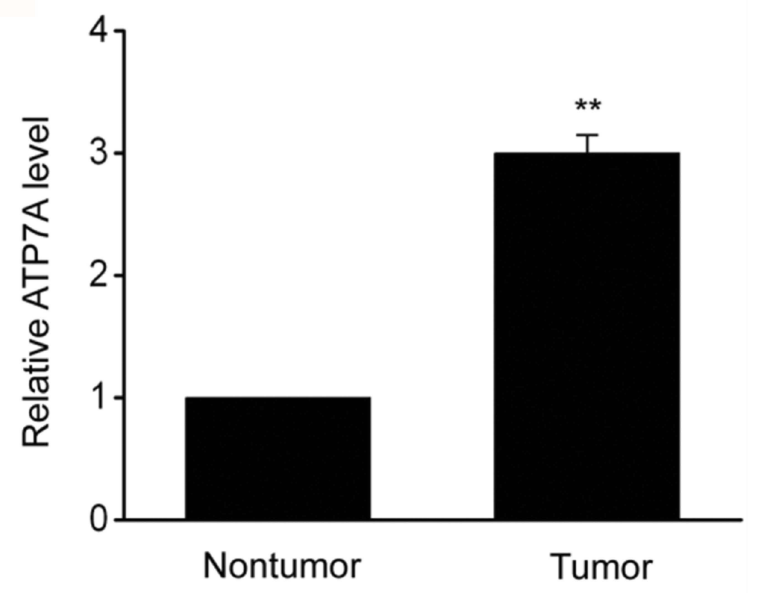

D

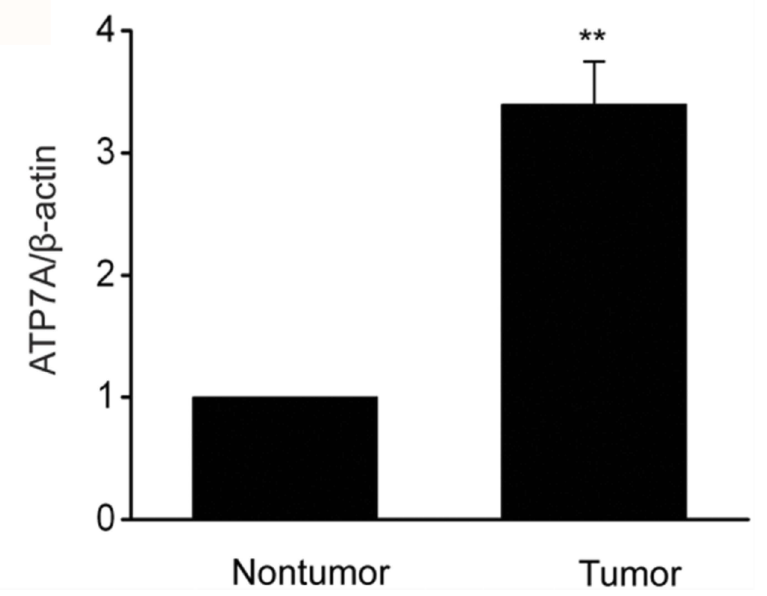

Figure I MiR-139was downregulated in OC specimens. (A,B) Expression levels of miR-I39 (A) and ATP7A (B) in OC specimens and in matched surrounding nonmalignant tissues were measured using qRT-PCR. C-D, Representative immunoblots (C) and quantitative assessment of ATP7A (D) in OC specimens and in matched surrounding non-malignant tissues. Results are displayed as means $\pm S E M, n=31, * * p<0.01$ vs tumor group. 
A

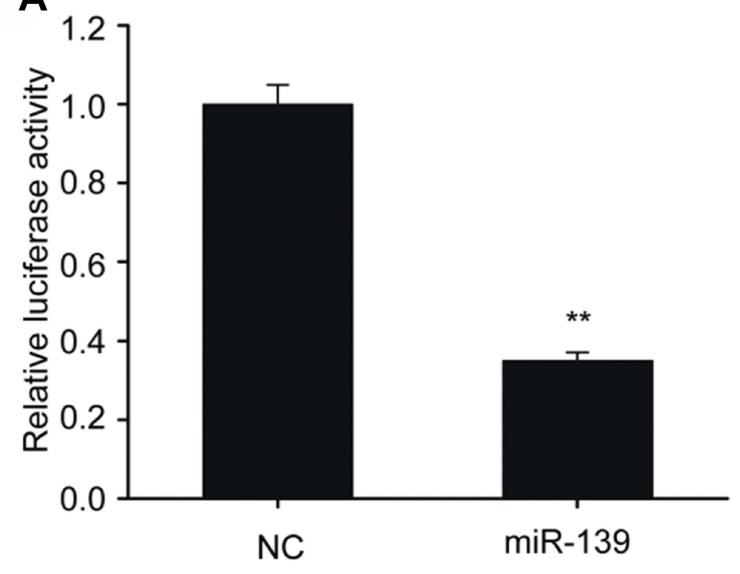

C

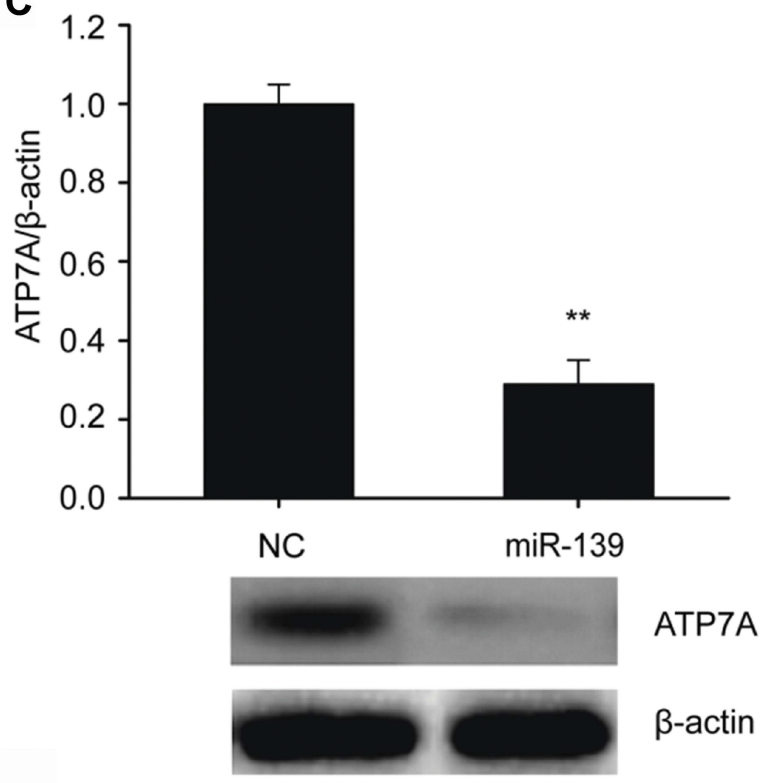

E

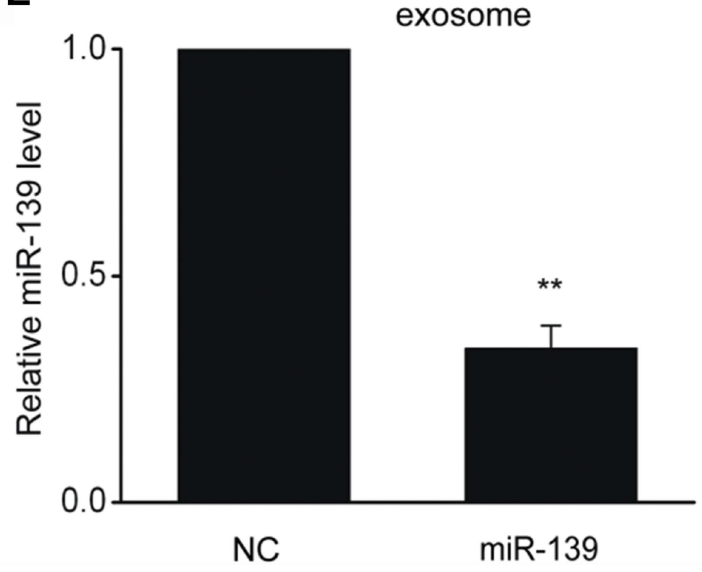

B

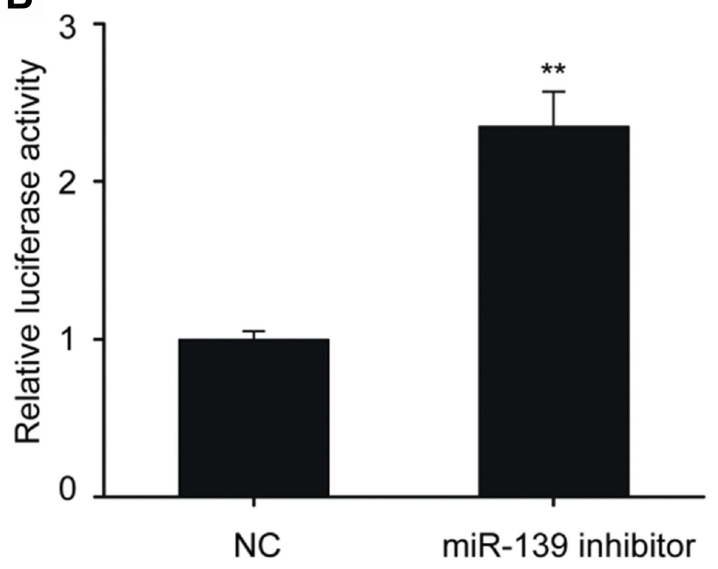

D
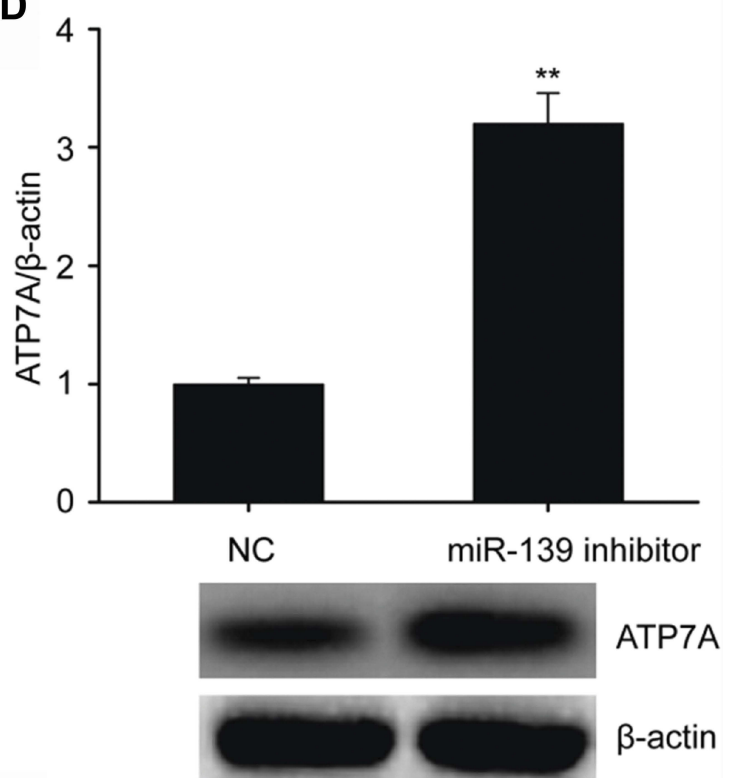

F
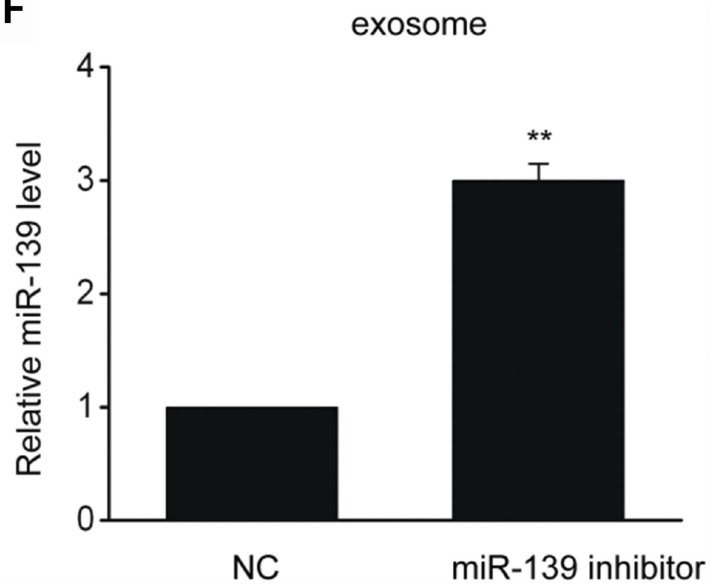

Figure 2 MiR-139 directly targeted ATP7A to impair exosome secretion-modulated miR-139 trafficking of SKOV3 cells. (A,B) Link between miR-I 39 and 3'-UTR of ATP7A mRNA was assessed by luciferase reporter assay. (C-D) Representative immunoblots and quantitative assessment of ATP7A in SKOV3 cells that underwent 48 -h transfection with miR-I39 (C) or miR-I39 inhibitor (D). (E-F) Expression of miR-139 in exosomes from SKOV3 cells that underwent 48-h transfection with miR-139 (E) or miR-I39 inhibitor (F) was assessed using qRT-PCR. Results are displayed as means \pm SEM, ${ }^{* * p} p 0.01$ vs NC group. 
translation in SKOV3 cells (Figure 2C and D). We isolated exosomes and examined miR-139 expression to explore the impact of the miR-139-ATP7A interaction on exosome secretion-modulated miR-139 trafficking. Excessive miR139 expression decreased miR-139 levels in exosomes (Figure 2E and F), but miR-139 inhibition increased it. These findings indicated that miR-139 directly targeted ATP7A to impair exosome secretion-modulated miR-139 trafficking in SKOV3 cells.

\section{miR-I39 Repressed Proliferation Of SKOV3 Cells}

SKOV3 cells were transfected with miR-139 mimics, then CCK8 and FC were performed to assess and explore the impact of miR-139 on OC proliferation. It was revealed via CCK8 that the proliferation of SKOV3 cells was remarkably repressed with excessive miR-139 expression compared to that of NC (Figure 3A). It was revealed via FC that the proportion of cells in $\mathrm{S}$ phase was lower in specimens transfected with miR-124 mimics than in those transfected with NC (Figure 3B). Furthermore, excessive miR-139 expression remarkably repressed colony generation of
SKOV3cells (Figure 3C and D). These findings suggested that miR-139 repressed proliferation of SKOV3 cells.

\section{miR-I39 Induced Death Of SKOV3 Cells}

FC was carried out with Annexin V/PI staining to evaluate the impact of miR-139 on cell death. Cells that were transfected with a miR-139 mimic displayed a remarkably elevated death rate in comparison to those of NC (Figure 4A and B). Proteins involved in cell death are crucial factors; thus, our research examined alterations in the expression of $\mathrm{Bax}$ and $\mathrm{Bcl} 2$ in SKOV3 cells. Bax was noticeably upregulated while Bcl 2 was remarkably downregulated in SKOV3 cells that were transfected with a miR-139 mimic compared to those in the NC (Figure 4C-E). These findings proved that miR-139 induced death of SKOV3 cells.

\section{miR-I39 Repressed Invasion And Migration Of SKOV3 Cells}

Our research subsequently explored the contribution of miR-139 to the invasion and migration of SKOV3 cells. Cell migration was assessed via migration test using transwell chambers without coating. It was revealed that

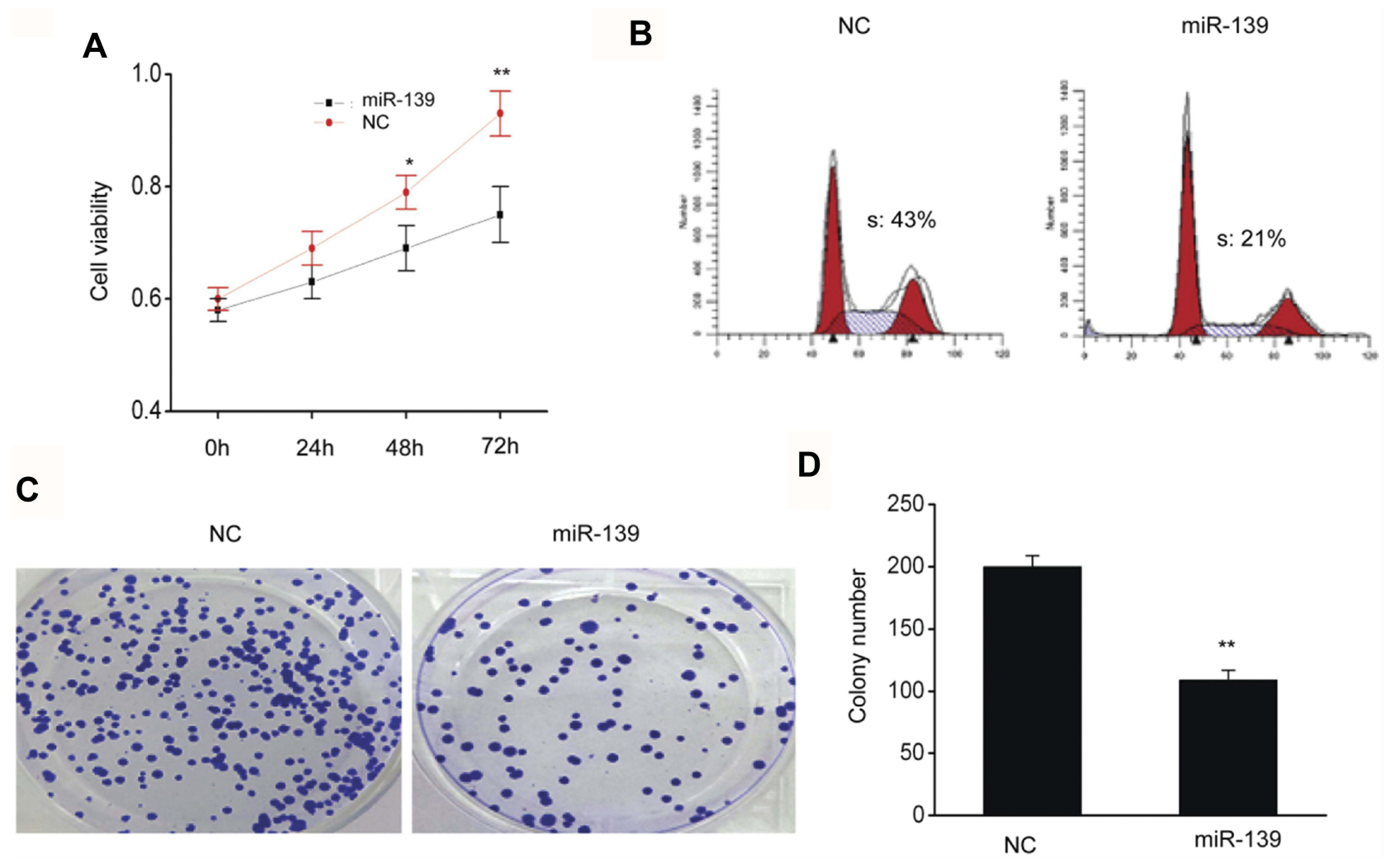

Figure 3 MiR-139 repressed the proliferation of SKOV3 cells. (A) MiR-139 repressed proliferation as demonstrated by CCK8 test. (B) MiR-I39 inhibited proliferation as demonstrated by FC. (C) The colonies were visualized after crystal violet staining. (D) Quantitative assessment of the number of colonies of SKOV3 cells that were transfected with NC or miR-139 mimics (miR-139). Results are displayed as means \pm SEM. ${ }^{*} p<0.05$, ${ }^{* *} p<0.01$ vs NC group. 

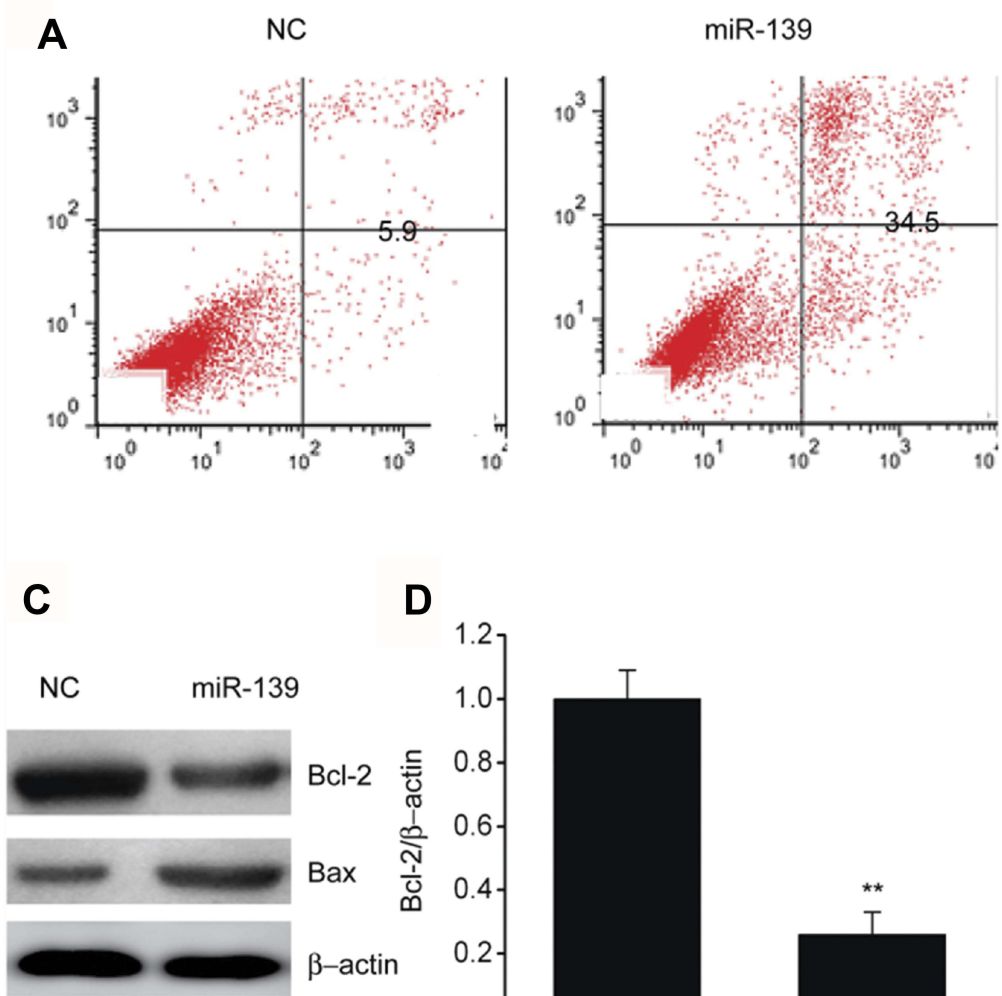

D

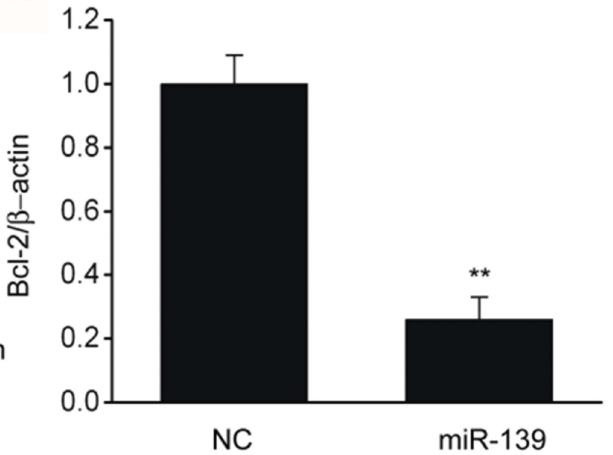

B

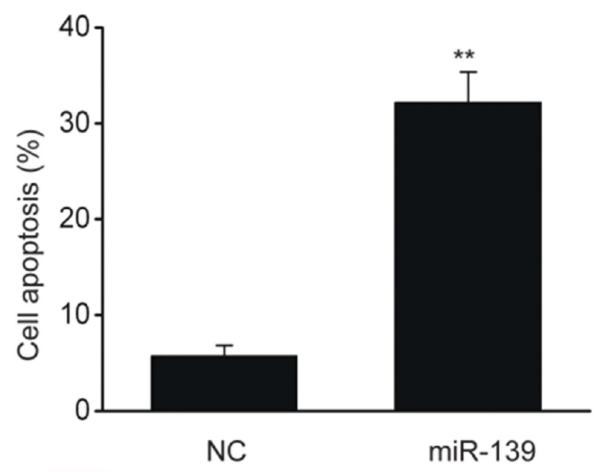

E

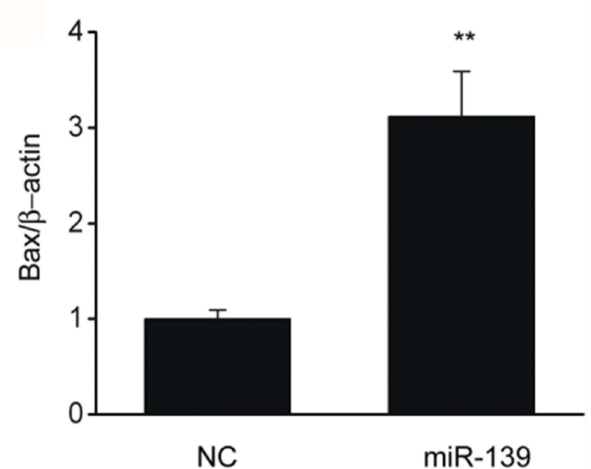

Figure 4 miR-139 inhibited death of SKOV3 cells. (A) Annexin V-PI FC was used to assess the death of SKOV3 cells. (B) Quantitative assessment of dead SKOV3 cells that were transfected with NC or miR-139 mimics (miR-139). C-E, Representative immunoblots (C) and quantitative assessment of Bcl2 (D) and Bax (E) in SKOV3 cells that were transfected with NC or miR-139 mimics (miR-139). Results are displayed as means \pm SEM. $*^{*} p<0.01$ vs NC group.

excessive miR-139 expression remarkably inhibited migration (Figure 5A and B). Cell invasion was assessed via invasion test using chambers with preliminary Matrigel coating. Invasion by cells transfected with miR-139 was less than those transfected with NC (Figure 5C and D). These findings suggested that miR-139 repressed the invasion and migration capabilities of SKOV3 cells.

\section{Excessive ATP7A Expression Counteracted Cell Growth Arrest And Migration Inhibition Induced By miR-I39 Mimics In SKOV3 Cells}

In SKOV3 cells transfected with miR-139 mimics, migration, proliferation, and cell death tests were carried out with or without pcDNA3-ATP7A to explore whether ATP7A was crucial for the impact of miR-139 on repressing malignancy in OC. Excessive ATP7A expression counteracted cell growth arrest and cell death induced by miR-139 mimics (Figure 6A and B). Furthermore, excessive ATP7A expression also counteracted migration inhibition induced by
miR-139 mimics (Figure 6C and 6D). These findings proved that ATP7A modulation was crucial to miR-139 in regulating cell death, migration, and proliferation of SKOV3 cells.

\section{miR-I39 Repressed OC Malignancy Growth In Vivo}

Male BALB/c nude mice were injected with SKOV3 cells in their flanks to verify whether miR-139 repressed OC growth. The malignancy volume and weight was remarkably lower and the expression of ATP7A in mice with excessive miR-139 expression than in those with the $\mathrm{NC}$ (Figure 7A-D). This in vivo finding indicated that miR139 repressed OC generation.

\section{Discussion}

Our research indicated that miR-139 expression was repressed in OC, bringing about excessive ATP7A expression and increasing cell death, proliferation as well as migration of OC cells. We revealed that miR-139 could modulate the generation and development of OC via the 
A

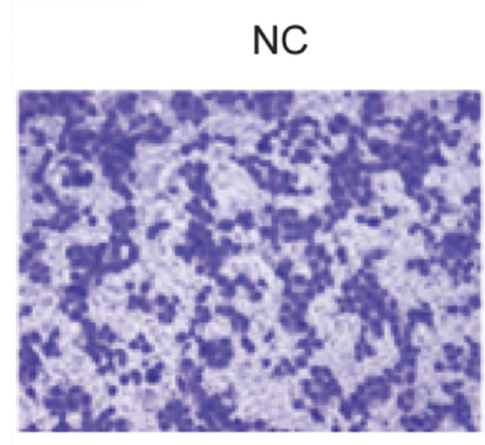

NC

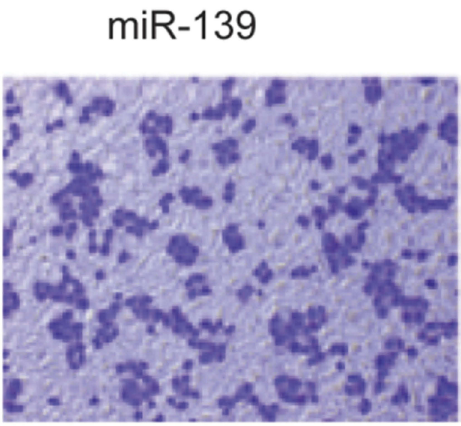

B

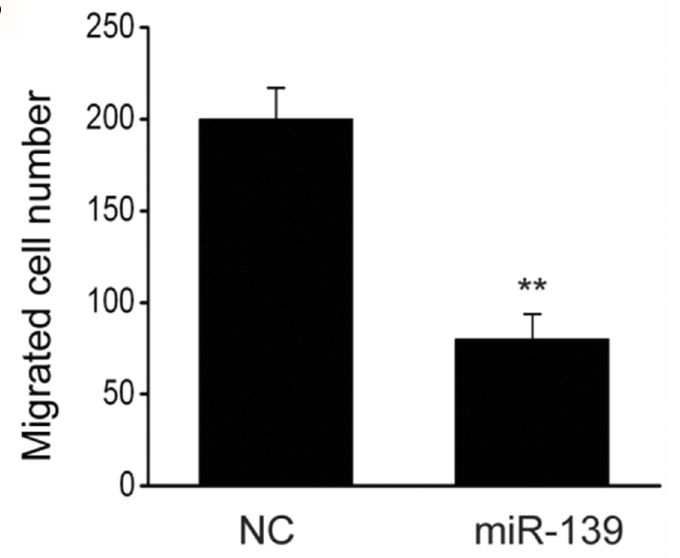

C

NC

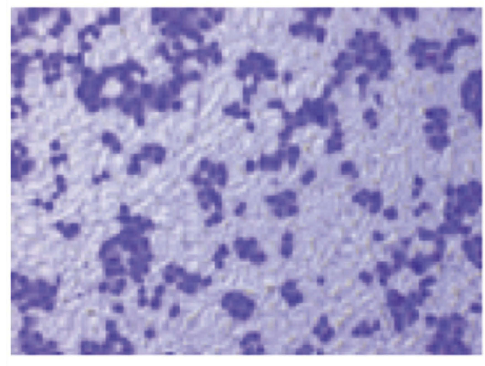

miR-139

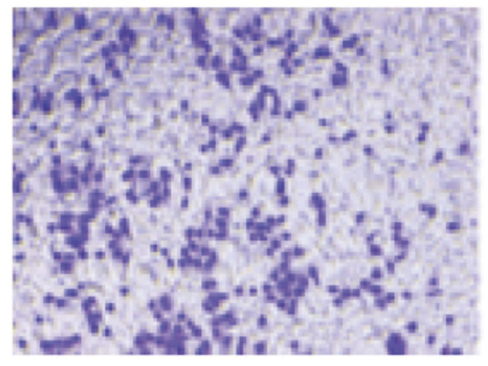

D

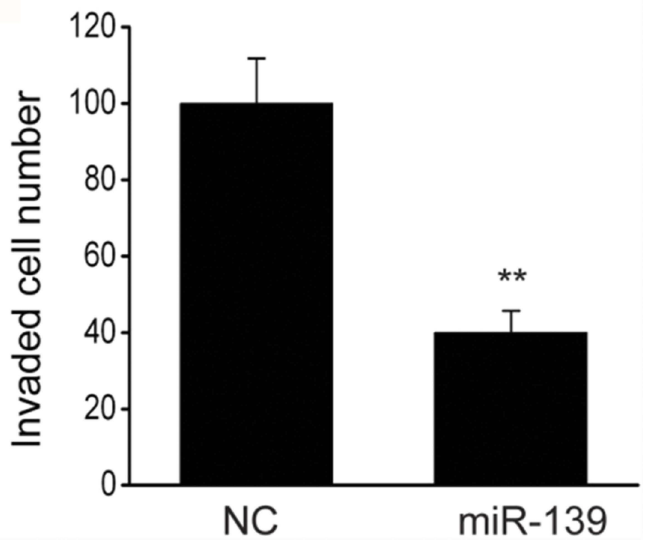

Figure 5 miR-139 inhibited OC cell invasion and migration. (A) Images revealing migrated SKOV3 cells transfected with NC or miR-I39 mimics (miR-I39) on the bottom surface of transwell membranes. (B) Number of migrated SKOV3 cells of various groups in 5 random fields under a microscope. (C) Images revealing invaded SKOV3 cells transfected with NC or miR-139 mimics (miR-139) on the bottom surface of transwell membranes. (D) Number of invaded SKOV3 cells of various groups in 5 random fields under a microscope. Results are displayed as means \pm SEM. ${ }^{* *} p<0.01$ vs NC group.

regulation of ATP7A. Our research sheds light on the impact of miR-139 on OC generation and indicates that miR-139/ATP7A could serve as a promising target for OC therapy.

Whether a certain miRNA is oncogenic or malignancyrepressing is mainly dictated by its target genes in various cellular reactions. ${ }^{23} \mathrm{MiR}-139$ has been previously found to repress some kinds of malignancies. ${ }^{24-26}$ MiR-139 targeted multiple crucial genes that contribute to motility. ${ }^{27,28} \mathrm{MiR}-$ 139-5p bound to NOTCH1 and AMFR mRNA, thus inhibiting the migration of cancer cells. ${ }^{29}$ Expression of miR-139 was repressed in glioblastoma, resulting in the inhibition of the migration of glioblastoma cells by targeting ZEB1. ${ }^{30}$ Furthermore, miR-139 expression was repressed in prostate cancer, resulting in the inhibition of migration and proliferation by targeting SOX5. ${ }^{31}$ Our study suggested that the expression of miR-139 was repressed in OC. Excessive miR-139 expression induced the death and inhibited the migration and proliferation of OC cells. Furthermore, in vivo findings indicated that excessive miR-139 expression remarkably impaired malignancy growth. These findings indicated that miR-139 exerted an essential malignancyrepressing impact on OC.

It has been demonstrated that miR-139 counteracts malignancy and represses proliferation and invasion by targeting various crucial genes. ${ }^{32}$ It was suggested via TargetScan that miR-139 targeted ATP7A. Some studies have indicated that ATP7A was upregulated and involved in the development of various malignancies, such as esophageal squamous cell cancer and breast cancer. ${ }^{33-35}$ A recent study revealed that ATP7A expression was upregulated and contributed to the invasion and proliferation of OC cells, ${ }^{33,36}$ 
A

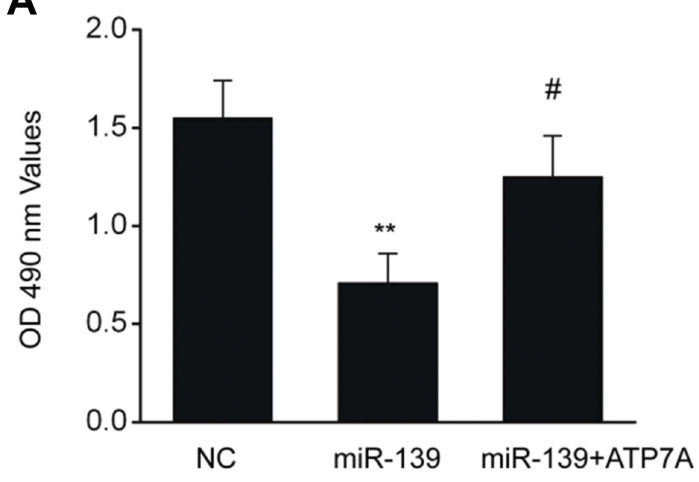

B

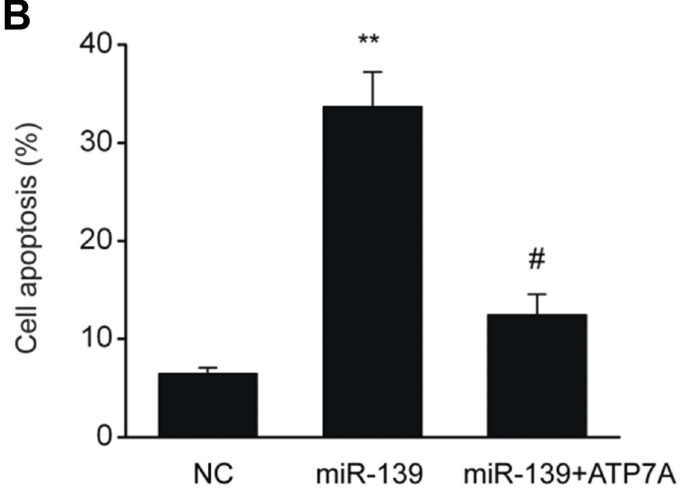

D

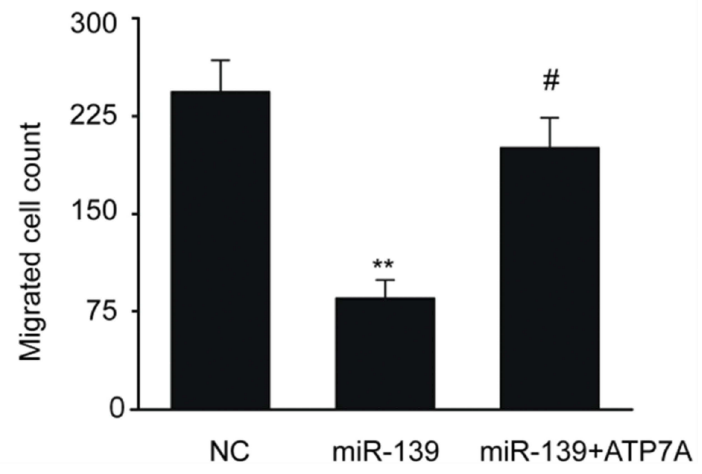

Figure 6 Excessive ATP7A expression counteracted the effects of miR-139 mimics. (A) Transfection of ATP7A counteracted cell growth arrest mediated by miR-139 mimics in SKOV3 cells. (B) Transfection of ATP7A counteracted cell death triggered by miR-139 mimics in SKOV3 cells. (C-D) Transfection of ATP7A counteracted migration prohibition triggered via miR-139 mimics in SKOV3 cells. Results are displayed in the form of means \pm SEM. ${ }^{* *} p<0.01$ vs NC group, ${ }^{\#} p<0.05$ vs miR-I39 group.

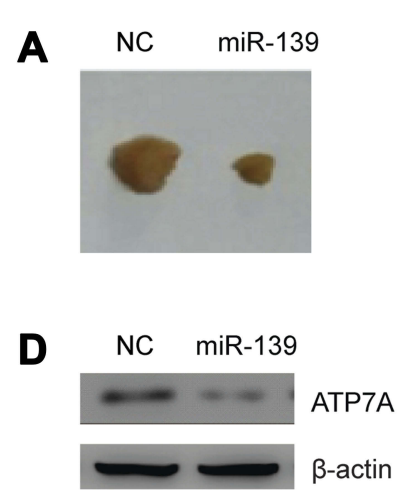

B

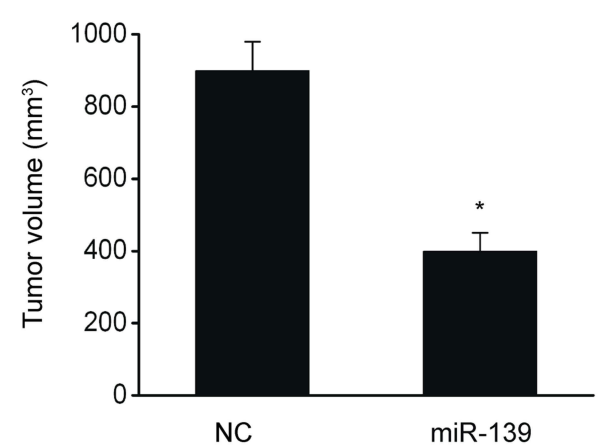

C

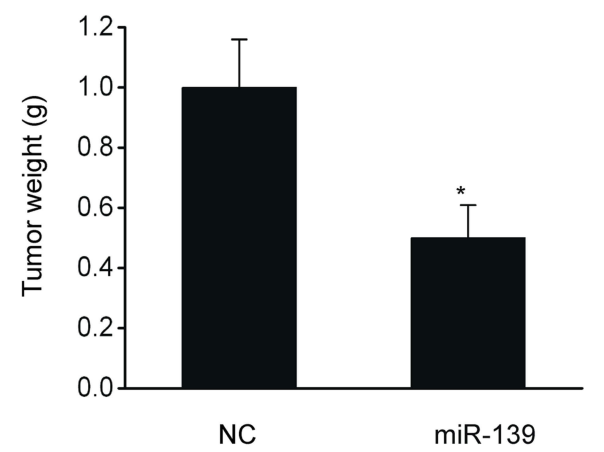

Figure 7 miR- 139 inhibited malignancy growth in vivo. (A) Photographs revealed malignancies in xenografts of SKOV3 cells with and without excessive miR-I39 expression. (B) Volume of the xenograft malignancy. (C) Weight of the xenograft malignancy. (D) Representative immunoblots of ATP7A in the xenograft. Results are displayed in the form of means \pm SEM. $n=6,{ }^{*} p<0.05$ vs NC group.

which counteracted the effect of miR-139. Our research proved through a luciferase reporter assay that miR-139 directly targeted ATP7A. Additionally, excessive miR-139 expression downregulated the transcription and translation of
ATP7A, while excessive ATP7A expression impaired miR139-mediated inhibition of proliferation and migration. Clinical samples showed that the expression of miR-139 was inversely related to that of ATP7A. 


\section{Conclusions}

In summary, this research indicated that miR-139 represses OC invasion and proliferation by targeting the expression of ATP7A. These findings could provide a potential innovative target for the treatment of OC.

\section{Ethics Approval And Informed Consent}

Fully informed consent was acquired from every participant, which won approval from Research Ethics Committee of Third Xiangya Hospital of Central South University (Changsha, China).

\section{Author Contributions}

All authors contributed to data analysis, drafting or revising the article, gave final approval of the version to be published, and agree to be accountable for all aspects of the work.

\section{Disclosure}

The authors declare that there are no conflicts of interest.

\section{References}

1. Gasiorowska E, Urbaniak B, Lorek J, Matysiak J, Nowak-Markwitz E. Proteomic pattern of cervico-vaginal fluid (CVF) in an ovarian cancer diagnosis - pilot study. Ginekol Pol. 2018;89(12):688-694. doi:10.5603/GP.a2018.0116

2. Halbur C, Choudhury N, Chen M, Kim JH, Chung EJ. siRNAconjugated nanoparticles to treat ovarian cancer. SLAS Technol. 2019;2472630318816668.

3. Mao W, Peters HL, Sutton MN, et al. The role of vascular endothelial growth factor, interleukin 8 , and insulinlike growth factor in sustaining autophagic DIRAS3-induced dormant ovarian cancer xenografts. Cancer. 2019;125(8):1267-1280. doi:10.1002/cncr.31935

4. Penet MF, Krishnamachary B, Wildes FB, et al. Ascites volumes and the ovarian cancer microenvironment. Front Oncol. 2018;8:595. doi:10.3389/fonc.2018.00595

5. Zhang Y, Cao L, Nguyen D, Lu H. TP53 mutations in epithelial ovarian cancer. Transl Cancer Res. 2016;5(6):650-663. doi:10.21037/tcr

6. Kuhlmann JD, Chebouti I, Kimmig R, et al. Extracellular vesicle-associated miRNAs in ovarian cancer - design of an integrated NGS-based workflow for the identification of blood-based biomarkers for platinum-resistance. Clin Chem Lab Med. 2019;57(7):1053-1062. doi:10.1515/cclm-2018-1048

7. Guo TY, Xu HY, Chen WJ, Wu MX, Dai X. Downregulation of miR-1294 associates with prognosis and tumor progression in epithelial ovarian cancer. Eur Rev Med Pharmacol Sci. 2018;22(22):7646-7652. doi:10.26355/eurrev_201811_16381

8. Luo L, Gao YQ, Sun XF. Circular RNA ITCH suppresses proliferation and promotes apoptosis in human epithelial ovarian cancer cells by sponging miR-10a-alpha. Eur Rev Med Pharmacol Sci. 2018;22 (23):8119-8126. doi:10.26355/eurrev_201812_16503

9. Prahm KP, Hogdall C, Karlsen MA, Christensen IJ, Novotny GW, Hogdall E. Identification and validation of potential prognostic and predictive miRNAs of epithelial ovarian cancer. PLOS ONE. 2018;13 (11):e0207319. doi:10.1371/journal.pone.0207319
10. Fu X, Tian J, Zhang $\mathrm{L}$, Chen $\mathrm{Y}$, Hao Q. Involvement of microRNA-93, a new regulator of PTEN/Akt signaling pathway, in regulation of chemotherapeutic drug cisplatin chemosensitivity in ovarian cancer cells. FEBS Lett. 2012;586(9):1279-1286. doi:10.101 6/j.febslet.2012.03.006

11. Guo FJ, Shao YP, Wang YP, Jin YM, Liu SS, Wang QY. MIR-92 stimulates VEGF by inhibiting von Hippel-Lindau gene product in epithelial ovarian cancer. J Biol Regul Homeost Agents. 2017;31 (3):615-624.

12. Mak CS, Yung MM, Hui LM, et al. MicroRNA-141 enhances anoikis resistance in metastatic progression of ovarian cancer through targeting KLF12/Sp1/survivin axis. Mol Cancer. 2017;16(1):11. doi:10. 1186/s12943-017-0582-2

13. Wang Y, Bao W, Liu Y, et al. miR-98-5p contributes to cisplatin resistance in epithelial ovarian cancer by suppressing miR-152 biogenesis via targeting Dicer1. Cell Death Dis. 2018;9(5):447. doi:10. 1038/s41419-018-0390-7

14. Bian Z, Zhang J, Li M, et al. Correction: long non-coding RNA LINC00152 promotes cell proliferation, metastasis, and confers 5 -FU resistance in colorectal cancer by inhibiting miR-139-5p. Oncogenesis. 2018;7(8):63. doi:10.1038/s41389-018-0067-1

15. Huang LL, Huang LW, Wang L, Tong BD, Wei Q, Ding XS. Potential role of miR-139-5p in cancer diagnosis, prognosis and therapy. Oncol Lett. 2017;14(2):1215-1222. doi:10.3892/ol.2017.6351

16. Jiang Y, Jiang J, Jia H, Qiao Z, Zhang J. Recovery of miR-139-5p in ovarian cancer reverses cisplatin resistance by targeting C-Jun. Cell Physiol Biochem. 2018;51(1):129-141. doi:10.1159/000495169

17. Ma J, Zhang J, Weng YC, Wang JC. EZH2-mediated microRNA-139$5 \mathrm{p}$ regulates epithelial-mesenchymal transition and lymph node metastasis of pancreatic cancer. Mol Cells. 2018;41(9):868-880. doi:10. 14348/molcells.2018.0109

18. Agosta C, Laugier J, Guyon L, et al. MiR-483-5p and miR-139-5p promote aggressiveness by targeting $\mathrm{N}$-myc downstream-regulated gene family members in adrenocortical cancer. Int J Cancer. 2018;143 (4):944-957. doi:10.1002/ijc.31363

19. Jiao W, Zhang J, Wei Y, et al. MiR-139-5p regulates VEGFR and downstream signaling pathways to inhibit the development of esophageal cancer. Dig Liver Dis. 2019;51(1):149-156. doi:10.1016/j. dld.2018.07.017

20. Sherafatian M. Tree-based machine learning algorithms identified minimal set of miRNA biomarkers for breast cancer diagnosis and molecular subtyping. Gene. 2018;677:111-118. doi:10.1016/j. gene.2018.07.057

21. Yong-Hao Y, Xian-Guo W, Ming X, Jin-Ping Z. Expression and clinical significance of miR-139-5p in non-small cell lung cancer. J Int Med Res. 2019;300060518815379.

22. Luo H, Yang R, Li C, et al. MicroRNA-139-5p inhibits bladder cancer proliferation and self-renewal by targeting the Bmi1 oncogene. Tumour Biol. 2017;39(7):1010428317718414. doi:10.1177 $/ 1010428317718414$

23. Song CJ, Chen H, Chen LZ, Ru GM, Guo JJ, Ding QN. The potential of microRNAs as human prostate cancer biomarkers: A meta-analysis of related studies. J Cell Biochem. 2018;119(3):2763-2786. doi:10. $1002 /$ jcb. 26445

24. Huang P, Xi J, Liu S. MiR-139-3p induces cell apoptosis and inhibits metastasis of cervical cancer by targeting NOB1. Biomed Pharmacother. 2016;83:850-856. doi:10.1016/j.biopha.2016.07.050

25. Pajic M, Froio D, Daly S, et al. miR-139-5p modulates radiotherapy resistance in breast cancer by repressing multiple gene networks of DNA repair and ROS defense. Cancer Res. 2018;78(2):501-515. doi:10.1158/0008-5472.CAN-16-3105

26. Wang Y, Li J, Xu C, Zhang X. MicroRNA-139-5p inhibits cell proliferation and invasion by targeting RHO-associated coiled-coilcontaining protein Kinase 2 in ovarian cancer. Oncol Res. 2018;26 (3):411-420. doi:10.3727/096504017X14974343584989 
27. Hua W, Sa KD, Zhang X, et al. MicroRNA-139 suppresses proliferation in luminal type breast cancer cells by targeting Topoisomerase II alpha. Biochem Biophys Res Commun. 2015;463(4):1077-1083. doi:10.1016/j.bbrc.2015.06.061

28. Zhang HD, Sun DW, Mao L, et al. MiR-139-5p inhibits the biological function of breast cancer cells by targeting Notch1 and mediates chemosensitivity to docetaxel. Biochem Biophys Res Commun. 2016;465(4):702-713. doi:10.1016/j.bbrc.2015.08.053

29. Liu H, Yin Y, Hu Y, et al. miR-139-5p sensitizes colorectal cancer cells to 5-fluorouracil by targeting NOTCH-1. Pathol Res Pract. 2016;212(7):643-649. doi:10.1016/j.prp.2016.04.011

30. Dai S, Wang X, Li X, Cao Y. MicroRNA-139-5p acts as a tumor suppressor by targeting ELTD1 and regulating cell cycle in glioblastoma multiforme. Biochem Biophys Res Commun. 2015;467 (2):204-210. doi:10.1016/j.bbrc.2015.10.006

31. Yang B, Zhang W, Sun D, et al. Downregulation of miR-139-5p promotes prostate cancer progression through regulation of SOX5. Biomed Pharmacother. 2019;109:2128-2135. doi:10.1016/j.biopha. 2018.09.029
32. Li Q, Liang $\mathrm{X}$, Wang $\mathrm{Y}$, et al. miR-139-5p inhibits the epithelial-mesenchymal transition and enhances the chemotherapeutic sensitivity of colorectal cancer cells by downregulating BCL2. Sci Rep. 2016;6:27157. doi:10.1038/srep27157

33. Li T, Peng J, Zeng F, et al. Association between polymorphisms in CTR1, CTR2, ATP7A, and ATP7B and platinum resistance in epithelial ovarian cancer. Int J Clin Pharmacol Ther. 2017;55(10):774-780. doi:10.5414/CP202907

34. Li ZH, Zheng R, Chen JT, Jia J, Qiu M. The role of copper transporter ATP7A in platinum-resistance of esophageal squamous cell cancer (ESCC). J Cancer. 2016;7(14):2085-2092. doi:10.7150/jca.16117

35. Zheng MD, Wang ND, Li XL, et al. Toosendanin mediates cisplatin sensitization through targeting Annexin A4/ATP7A in non-small cell lung cancer cells. J Nat Med. 2018;72(3):724-733. doi:10.1007/ s11418-018-1211-0

36. Xiao F, Li Y, Wan Y, Xue M. MircroRNA-139 sensitizes ovarian cancer cell to cisplatin-based chemotherapy through regulation of ATP7A/B. Cancer Chemother Pharmacol. 2018;81(5):935-947. doi:10.1007/s00280-018-3548-1

\section{Publish your work in this journal}

OncoTargets and Therapy is an international, peer-reviewed, open access journal focusing on the pathological basis of all cancers, potential targets for therapy and treatment protocols employed to improve the management of cancer patients. The journal also focuses on the impact of management programs and new therapeutic agents and protocols on patient perspectives such as quality of life, adherence and satisfaction. The manuscript management system is completely online and includes a very quick and fair peer-review system, which is all easy to use. Visit http://www.dovepress.com/ testimonials.php to read real quotes from published authors. 